\section{How to induce ovarian cancer: and how not to}

One of the most elegant subterfuges in experimental carcinogenesis was to transplant ovaries from the pelvis to a site with portal drainage and induce a high incidence of ovarian tumours-all without benefit of carcinogens. The pituitary had to be intact. With the changed ovarian position oestrogens in the venous blood going directly to the liver were inactivated before they could exert feedback on the pituitary, thus allowing an uninhibited production of gonadotrophins. ${ }^{12}$ In addition to showing that gonadotrophins could play a part in producing ovarian cancer these findings from research in animals cast oestrogens in the unusual role of protectors against cancer. Unfortunately, the tumours were of stromal origin which are neither common nor highly malignant in man; so, apart from admiration for the ingenuity underlying the model, little attention was paid to the "gonadotrophin hypothesis" which evolved from it. Recently, however, these old findings have been re-examined as possibly relevant to the surface epithelial cancers ${ }^{3}$ which make up most cases of ovarian cancer ${ }^{+}$and make it the greatest gynaecological cause of mortality-3500 deaths a year in England and Wales. ${ }^{56}$

A feature of ovarian cancer is that the exponential increase in mortality rate with age found with most epithelial malignancies disappears at around 65: a plateau covers the more advanced age groups. ${ }^{7}$ A similar pattern is seen in the incidence of breast cancer, which suggests that for both these cancers some important aetiological factor stops operating in later life. ${ }^{8}$ Given the likely time lag before any effect is reflected in mortality, some process that ends at about the end of the fifth decade, possibly related to the ovulatory cycle, seems a likely candidate.

Ovarian cancer is a disease of developed countries in which limitation of family size is usual. ${ }^{9}$ Many studies have shown that pregnancies confer an outstanding degree of protection and (conversely) that women who for whatever reason never become pregnant have a raised incidence of the disease. Early age at the time of the first pregnancy may be of particular importance. ${ }^{10}$ Other factors which have been implicated range from family history, radiation, asbestos, hysterectomy, age at menarche and menopause, blood group, consumption of non-contraceptive hormones, diet, and mumps virus to applying talc to the external genitals. ${ }^{7}$ None of these, however, seems likely to be a major influence. The only protective factor which matches pregnancy in the strength of its effect is the use of oral contraceptives, " which has been estimated to prevent 1700 cases a year in the United States. ${ }^{12}$

How do pregnancy and the "pill" exert their protective effects? The most obvious common factor is that both suppress ovulation. Fathalla first advanced the idea that incessant ovulation was the "bad habit," which if allowed to persist built up the risk of ovarian cancer. ${ }^{13}$ This line of thought developed against a background of knowledge that domestic fowls bred for maximal egg laying frequently had ovarian cancer-and that those gullible fowls persuaded by manipulation of the light in their environment that springtime was forever and so kept up a frenzy of egg laying had a very high incidence. Since then much evidence has appeared to support Fathalla's idea, particularly the fact that anovulant contraceptive usage is consistently associated with a reduced risk.
The problem is that some of the facts seem too good to fit the hypothesis. For example, a woman who has one pregnancy and breast feeds may be estimated to miss about $3 \%$ of the ovulations which would have occurred if she had never been pregnant: but a single pregnancy is associated with a reduction in incidence by about half. ${ }^{7}$ Data on oral contraceptives point to a strong effect even with short periods of use persisting long after this has stopped ${ }^{14}$ : the mean impact recorded in a review of 10 studies was a reduction of $40 \% . "$ Though controversy still surrounds the "pill"- and probably always will-its unexpected benefits need to be assessed with care so that a balanced view can be obtained of the undesirable side effects, which otherwise receive all the attention.

The recent resurrection of the gonadotrophin hypothesis came in one of a series of papers resulting from a big study in Boston, Massachusetts ${ }^{15-18}$ - though the findings probably do not justify the conclusions drawn. Like others before, the authors appear to have been attracted to the ingenious animal model. They say that: "the gonadotrophin hypothesis is appealing in that it allows inferences to be drawn from the animal models concerning human ovarian neoplasia." The fact that the graph of ovarian cancer plateaus rather than continues to rise after the time of the menopause must be considered against the gonadotrophin hypothesis as at this time there are sustained high concentrations. That said, however, the cyclic gonadotrophins have an essential role in the production of steroids every month and any new slant may cast light on the problem of how ovulation seems to affect the incidence of ovarian cancer. The impact of ovulation is often assessed on the basis of estimated "ovulatory age" - starting at menarche and allowing for pregnancies, lactation, and so on. ${ }^{19}$ The Boston group ignored this approach-which is rather like doing an epidemiological study of lung cancer and choosing to exclude consideration of the number of cigarettes smoked. ${ }^{17}$ They speculated that pregnancy may have a permanent effect on the pituitary secretion of trophic hormones. This is based on rather tenuous anatomical grounds, but nevertheless the evidence does point to some such semipermanent resetting of a homoeostatic control mechanism.

There are striking similarities and differences in the backgrounds of ovarian and breast cancers. The differences are probably attributable to differing hormone sensitivities in the tissues concerned. At a recent symposium in Bruges of the European Organisation for Co-operation in Cancer Prevention Studies Professor Malcolm Pike suggested that the explanation for the impact of child bearing on breast cancer could be the sustained rise in the concentration of sex hormone binding globulin after pregnancy, ${ }^{8}$ and this might also account for the effect on ovarian cancer. Hormonal contraceptive regimens induce a similar effect-and this may explain benefits extending beyond the period of hormone consumption.

JAMES S SCOTT

Professor of Obstetrics and Gynaecology,

University of Leeds,

Leeds LS2 9NS

I Biskind MS, Biskind GR. Development of tumors in the rat ovary after transplantation into the spleen. Proc Soc Exp Biol Med 1944;55:176-9.

Biskind GR, Biskind MS. Atrophy of ovaries transplanted to the spleen in unilaterally castrated rats; proliferative changes following subsequent removal of intact ovary. Science 1948; 108:137-8.

3 Cramer DW, Welch WR. Determinants of ovarian cancer risk. II. Inferences regarding pathogenesis. FNCI 1983; 71:717-21.

4 Beller U, Bigelow B, Beckman EM, Brown B, Demopoulos RI. Epitheliat carcinoma of the ovary in the reproductive years: clinical and morphological characterization. Gynecol Oncol

5 Office of Population Censuses and Surveys. Cancer statistics: registrations. 1983a. London: HMSO, 1983. (Series MB1, No 11.) 
6 Office of Population Censuses and Surveys. Mortality statistics: cause. 1983b. London: HMSO, 1983. (Series DH2, No 9.)

its occurrence and aetiology. In: Bleehan N, ed. Recent results in cancer research -ovarian cancer. Heidelberg, New York: Springer-Verlag (in press). Hormones and sexual factors in human cancer aetiology. Proceedings of 2nd annual European Organisation for Co-operation in Cancer Prevention Studies symposium, i984. Amsterdam Elsevier (in press).

9 Lingeman CH. Environmental factors in the etiology of carcinoma of the human ovary: a review. Am f Ind Med 1983;4:365-79.

10 La Vecchia C, Franceschi S, Liberati A, Gallus G, Tognoni G. The clinical relevance of the epidemiology of ovarian cancer. European fournal of Clinical Oncology 1984;20:175-82

Beral V, Franceschi S. The prevention of ovarian cancer. In: Scott JS, Wolff JP, eds. Hormones and sexual factors in human cancer aetiology. Proceedings of 2 nd annual European Organisation for Co-operation in Cancer Prevention Studies symposium, 1984. Amsterdam. Elsevier (in press). the risk of ovarian cancer. FAMA 1983;249:1596-9.

the risk of ovarian cancer. $f A M A$ 1983;249:1596-9.

4 Rosenberg L, Shapiro S, Slone D, et al. Epithelial ovarian cancer and combination oral contraceptives. $\mathcal{F} A M A$ 1982;247:3210-2.

Cramer DW, Hutchison GB, Welch WR, Scully RE, Knapp RC. Factors affecting the association of oral contraceptives and ovarian cancer. $N$ Engl $\mathcal{Y}$ Med 1982;307:1047-51.

Cramer DW, Welch WR, Scully RE, Wojciechowski CA. Ovarian cancer and talc: a case-control

study. Cancer 1982;50:372-6.
Cramer DW, Hutchison GB, Welch WR, Scully RE, Ryan KJ. Determinants of ovarian cancer risk. I. Reproductive experiences and family history. ${ }^{2} N C I$ 1983;71:711-6.

8 Cramer DW, Welch WR, Cassalls S, Scully RE. Mumps, menarche, menopause and ovarian cancer. Am f Obstet Gynecol 1983;147:1-6. Casagrande JT, Louie EW, Pike MC, Roy S, Ross RK, Henderson BE. "Incessant ovulation"

\section{At least one centimetre for each millimetre}

The width of the excision margin for cutaneous melanoma remains a matter for debate. Since the pioneer description by Handley of the findings at necropsy in a patient with malignant melanoma with extensive spread of the tumour along the fascial planes around the primary lesion, ${ }^{12}$ wide three dimensional surgery has been the vogue. ${ }^{34}$ Nevertheless, Breslow and Macht challenged the view that a $5 \mathrm{~cm}$ margin of healthy tissue should be removed with all melanomas, finding that patients with superficial melanomas had an excellent survival irrespective of the size of the resection margin. ${ }^{5}$ Since then many workers have agreed that the loss of a large area of skin is unnecessary for tumours of limited thickness. ${ }^{6-9}$

Should, then, we excise all melanomas with a narrow margin? Ackerman and Scheiner have emphasised that it is sufficient to extirpate the primary lesion and little more than that; if histological assessment confirms that the resection margins are free of tumour then no further local surgery is warranted. ${ }^{10}$ Thus, they contend, surgery for cutaneous melanoma should be no different from that for other primary tumours in the skin. Nevertheless, their reasoning warrants further scrutiny. The biological behaviour of melanoma is distinctive in its tendency to form satellite and in transit metastases in the skin around the primary lesion or in the region between the primary lesion and the lymph nodes. Such metastases usually surface near the primary melanoma and subsequently spread over the entire regional area. Wide local excision aims at the eradication not only of the primary tumour but also of any occult satellite lesion in its immediate vicinity.

The importance of satellites in the management of melanoma should not be underestimated. Although clinically apparent in a minority of cases, satellite lesions are common microscopically and their prevalence is related to the thickness of the tumour. ${ }^{11}$ Day et al found no microscopic satellites at all in lesions of under $1 \mathrm{~mm}$ thick, but these were present in almost two thirds of lesions over $4 \mathrm{~mm}$ thick. " Similarly, Elder et al found that satellites and local recurrences occurred only in melanomas over $2 \mathrm{~mm}$ thick. ${ }^{12}$ It seems reasonable, therefore, to tailor the extent of local surgery to the thickness of the tumour.
Yet the published studies have failed to show that wide excision improves patient survival. ${ }^{5812}$ The two largest published series show a striking increase in the frequency of "local" failure with narrower margins. ${ }^{89}$ In the World Health Organisation study the rate of local recurrence was about three times higher in patients whose tumours had been excised with narrow tissue margins than in those subjected to wide excisions, irrespective of the thickness of the tumour. ${ }^{813}$ Similarly, in the Munich series the local recurrence rate was $10 \%$ for cases with margins of $3 \mathrm{~cm}$ or less and $3 \%$ for those with margins of more than $3 \mathrm{~cm} .{ }^{9}$ In both studies the differences were statistically significant. Generally local recurrence presages a dismal outcome, a fact which has been appreciated since the days of $\mathrm{McNeer},{ }^{14}$ yet in these series patients' ultimate survival was not substantially affected by the width of the excision margin. This paradox may be due to the heterogeneity of the various subsets, to the use of single factor statistical analyses instead of proportional hazards regression models, or to the follow up periods having been too short.

If we can prevent local recurrence by wide excision we should consider the procedure in each case, but especially for thick lesions. Many dermatologists and surgeons who have grown up in the era of safe margins of excision will hesitate to practise the new vogue of minimal procedures. ${ }^{10}$ The removal of an extra margin of ostensibly normal skin appears justified in high risk cases to remove any tumour satellite that may be present. Day et al recommend a $1.5 \mathrm{~cm}$ margin for low risk melanomas and a $3 \mathrm{~cm}$ margin for the high risk ones, dictated by the thickness and location of the primary tumour. ${ }^{15}$ These authors suggest that, because of their poor prognosis, BANS lesions (upper back, posterior upper $a \mathrm{rm}$, posterior neck, posterior scalp) ${ }^{16}$ should be excised with a $3 \mathrm{~cm}$ margin when they are of an intermediate thickness; for non-BANS melanomas of intermediate thickness an excision of $1.5 \mathrm{~cm}$ of clinically normal skin is adequate. Nevertheless, the BANS concept has recently been challenged. ${ }^{17}$

Whether localisation as such is a criterion for the width of the excision margin still needs further study. Until we have more data we should not rely on minimal resection margins. Only in very thin melanomas ( $\leqslant 1 \mathrm{~mm}$ ) should the margin be $1 \mathrm{~cm}$; in the intermediate thickness range $(1-2 \mathrm{~mm})$ a margin of $2-3 \mathrm{~cm}$ may be necessary; and for thick lesions $(>2 \mathrm{~mm}$ ) a $3-5 \mathrm{~cm}$ border should be excised so long as this does not include important structures. The clinician has the far from easy task of navigating between Scylla and Charybdis: on the one hand, increasing tumour thickness necessitates wider surgical excision; on the other, cosmetic and functional considerations may demand that this margin is smaller.

F H J RAMPEN

Consultant Dermatologist,

Academisch Medisch Centrum,

1105 AZ Amsterdam,

The Netherlands

1 Handley WS. The pathology of melanotic growths in relation to their operative treatment; I. Lancet 1907; i:927-33.

Handley WS. The pathology of melanotic growths in relation to their operative treatment; II Lancet $197,1.996-1003$

, Malignant melanomas of the skin; a study of the origin, development, aetiology, spread, treatment and prognosis. Br J Plast Surg 1962;15:

The surgical therapy of malignant melanomas. Semin Oncol 1975;2:175-8. Breslow A, Macht SD. Optimal size of resection margin for thin cutaneous melanoma. Surg Gynecol Obstet 1977;145:691-2.

6 Balch CM, Murad TM, Soong S-J, Ingalls AL, Richards PC, Maddox WA. Tumor thickness as a guide to surgical management of clinical stage I melanoma patients. Cancer 1979;43:883-8. Bagley FH, Cady B, Lee A, Legg MA. Changes in clinical presentation and management of

malignant melanoma. Cancer 1981;47:2126-34.
Cascinelli N, Van der Esch EP, Breslow A, Morabito A, Bufalino R. Stage I melanoma of the skin; the problem of resection margins. Eur J Cancer 1980;16:1079-85. 